\title{
Second-line docetaxel-based chemotherapy after failure of fluorouracil-based first-line treatment for advanced esophageal squamous cell carcinoma
}

This article was published in the following Dove Press journal:

OncoTargets and Therapy

13 October 2014

Number of times this article has been viewed

\section{Zhengbo Song \\ Yiping Zhang}

Department of Chemotherapy,

Zhejiang Cancer Hospital, Hangzhou,

People's Republic of China
Correspondence: Yiping Zhang Department of Medical Oncology,

Zhejiang Cancer Hospital, 38 Guangji Road 3 I 0022, Hangzhou, People's

Republic of China

$\mathrm{Tel}+8657188122188$

$\mathrm{Fax}+8657188122182$

Email zjzlyy16@।63.com
Purpose: This retrospective analysis evaluates the clinical efficacy and toxicity of second-line docetaxel-based chemotherapy after failure of fluorouracil-based first-line treatment for advanced esophageal squamous cell carcinoma (ESCC).

Methods: We retrospectively reviewed patients who had received second-line docetaxelbased chemotherapy for advanced ESCC in Zhejiang Cancer Hospital between January 2008 and December 2011. Survival curves were plotted using the Kaplan-Meier method. The Cox proportional hazard model was used for multivariate analysis.

Results: Eighty-five patients received docetaxel-based second-line chemotherapy after the failure of first-line fluorouracil-based treatment. Forty-four patients received docetaxel-platinum chemotherapy, and 41 received docetaxel single-agent treatment. The progression-free survival (PFS) and overall survival (OS) were 3.5 and 5.5 months in all of the patients, respectively. There were no statistically significant differences in PFS and OS between docetaxel-platinum and docetaxel single-agent chemotherapy groups ( $P$-value 0.38 and 0.64 , respectively). Response to first-line chemotherapy was a favorable prognostic factor for PFS in uni- and multivariate analyses ( $P$-value 0.005 and 0.028 , respectively).

Conclusion: Patients with docetaxel-based second-line treatment obtained a moderate PFS advantage in advanced ESCC. Response to first-line chemotherapy was a favorable prognostic factor for PFS of second-line chemotherapy in advanced ESCC.

Keywords: ESCC, efficacy, toxicity

\section{Introduction}

Esophageal carcinoma is one of the most common malignancies worldwide. ${ }^{1}$ The survival rate at 5 years is about $10 \%$ in Western countries, and worse in developing countries. ${ }^{2}$ Esophageal squamous cell carcinoma (ESCC) has historically been the most common histology. Palliative chemotherapy is the only option for patients with advanced esophageal cancer. Several palliative chemotherapy regimens have been investigated and have shown efficacy as first-line treatment. ${ }^{3}$ A combination of continuous-infusion 5-fluorouracil and cisplatin is the most commonly used regimen, with approximately a 30\% response rate in advanced esophageal carcinoma in current clinical practice. ${ }^{4}$ However, data on second-line therapy are scarce, and there has been no consensus on the optimal second-line chemotherapy until now.

Docetaxel exhibits broad-spectrum anticancer activity. Recently, docetaxel has been used as second-line chemotherapy in esophagogastric adenocarcinoma and demonstrated good efficacy. ${ }^{5}$ However, the study evaluation of docetaxel as second-line treatment of ESCC is limited; data on no more than 200 patients have been reported. ${ }^{6}$ 
We conducted an analysis in Chinese populations to evaluate efficacy and toxicity of docetaxel-based therapy as secondline chemotherapy of ESCC patients who did not respond to first-line fluorouracil-based treatment.

\section{Materials and methods Patients}

A clinical review of patients at Zhejiang Cancer Hospital between January 2008 and December 2011 was conducted. Esophageal carcinoma staging was performed according to the American Joint Committee on Cancer tumor-node-metastasis staging system published in 2009. Inclusion criteria consisted of: 1) Pathologically or cytologically proven primary ESCC, 2) patients received fluorouracil-based as a first-line treatment, 3) disease recurrence was confirmed using a chest/ abdomen computed tomography (CT) and bone scan as well as an ultrasound examination, 4) no local treatment like radiotherapy therapy was used during the period of secondline treatment, 5) patients had at least one measurable lesion and an Eastern Cooperative Oncology Group performance status of zero to two.

\section{Responses and toxicity evaluation}

Tumor responses were assessed with a CT scan at 6-week intervals until the lesions were evaluated as a progressive disease. Response Evaluation Criteria In Solid Tumors (RECIST) 1.1 was used to evaluate tumor response. The toxicity of treatment was evaluated in accordance with the National Cancer Institute Common Toxicity Criteria (NCI-CTC) 3.0.

\section{Follow-up}

All the patients that were evaluated for the second-line chemotherapy had progression-free survival (PFS). No patients were lost to follow-up. The median follow-up period was 10.2 months (3.9-26 months). The last follow-up date was December 31, 2013.

\section{Statistical analysis}

PFS encompassed the time from the second-line therapy to documented progression or death from any cause. Survival was recorded from the initiation of second-line treatment to the date of death or that of the last follow-up visit. The survival curves were calculated according to the KaplanMeier method. The Cox proportional model was used to evaluate various prognostic factors. Analyses were conducted using the computer software SPSS version 17.0 (SPSS Inc., Chicago, IL, USA).

\section{Results \\ Patient characteristics}

Overall, 1,255 patients were diagnosed with primary ESCC between January 2008 and December 2011. Of these patients, 667 had a recurrence after surgery or advanced disease. Firstline therapy was administered to 569 patients, 401 of which received fluorouracil-based first-line treatment. Of these 401 patients, 241 patients received second-line or further treatment. Clinical efficacy and toxicity evaluations could be conducted on 222 patients. Among these 222 patients, 85 patients received docetaxel-based chemotherapy.

Among the 85 patients who received docetaxel-based treatment, PS 0-1 was present in 54 patients (63.5\%) and PS 2 accounted for $36.5 \%$. Sixty patients were at an advanced stage on presentation, and 25 were experiencing a disease recurrence. Forty-four patients received docetaxel-platinum treatment in second-line treatment, 41 with docetaxel single-agent treatment. The baseline characteristics of patients are listed in Table 1.

\section{Efficacy analyses}

Among the 85 ESCC patients, 21 had partial response (PR) and 27 had stable disease (SD) in second-line treatment,

Table I Demographic characteristics of the study population

\begin{tabular}{ll}
\hline & Number \\
\hline Sex & 79 \\
Male & 6 \\
Female & \\
Age & 3 I-78 \\
Range & 56 \\
Median & 57 \\
$<60$ & 28 \\
$\geq 60$ & \\
ECOG PS & 54 \\
0-I & 31 \\
2 & \\
Initial disease status & 65 \\
Metastatic & 20 \\
Relapsed & \\
Metastatic sites & 25 \\
Lymph node & 20 \\
Lung & 20 \\
Liver & 12 \\
Bone & 8 \\
Others & \\
Response to first-line treatment & \\
CR + PR & 40 \\
SD + PD & 45 \\
Chemotherapy regimen & \\
Docetaxel-platinum & 44 \\
Docetaxel & 41 \\
\hline Abreviatons CR comple respons; ECOG PS & \\
\hline & \\
\hline
\end{tabular}

Abbreviations: CR, complete response; ECOG PS, the Eastern Cooperative Oncology Group scale of Performance Status; PD, progressive disease; PR, partial response; SD, stable disease. 


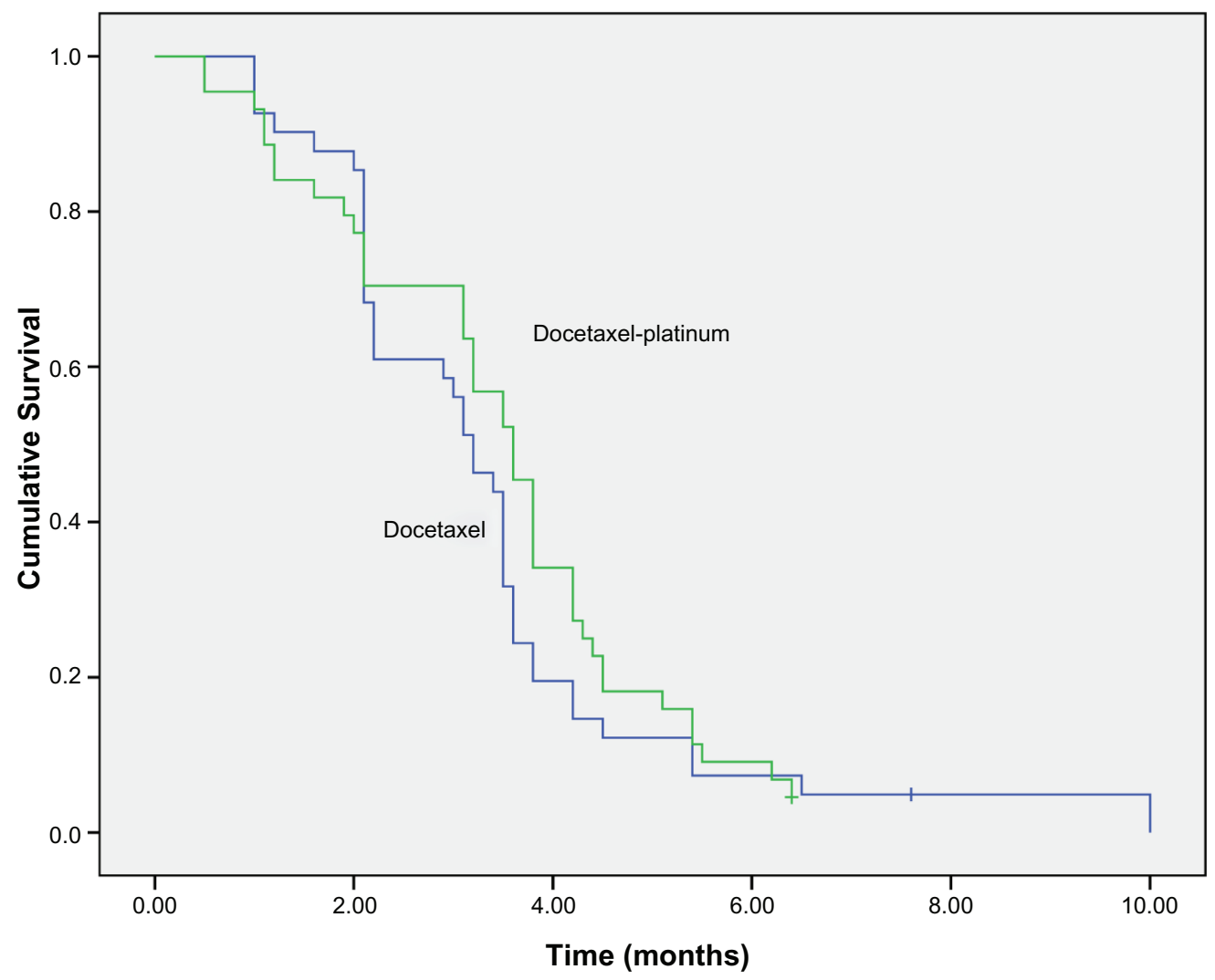

Figure I PFS in docetaxel-platinum and docetaxel single-agent treatments group ( 3.6 vs 3.2 months, $P=0.38$ ).

Abbreviation: PFS, progression-free survival.

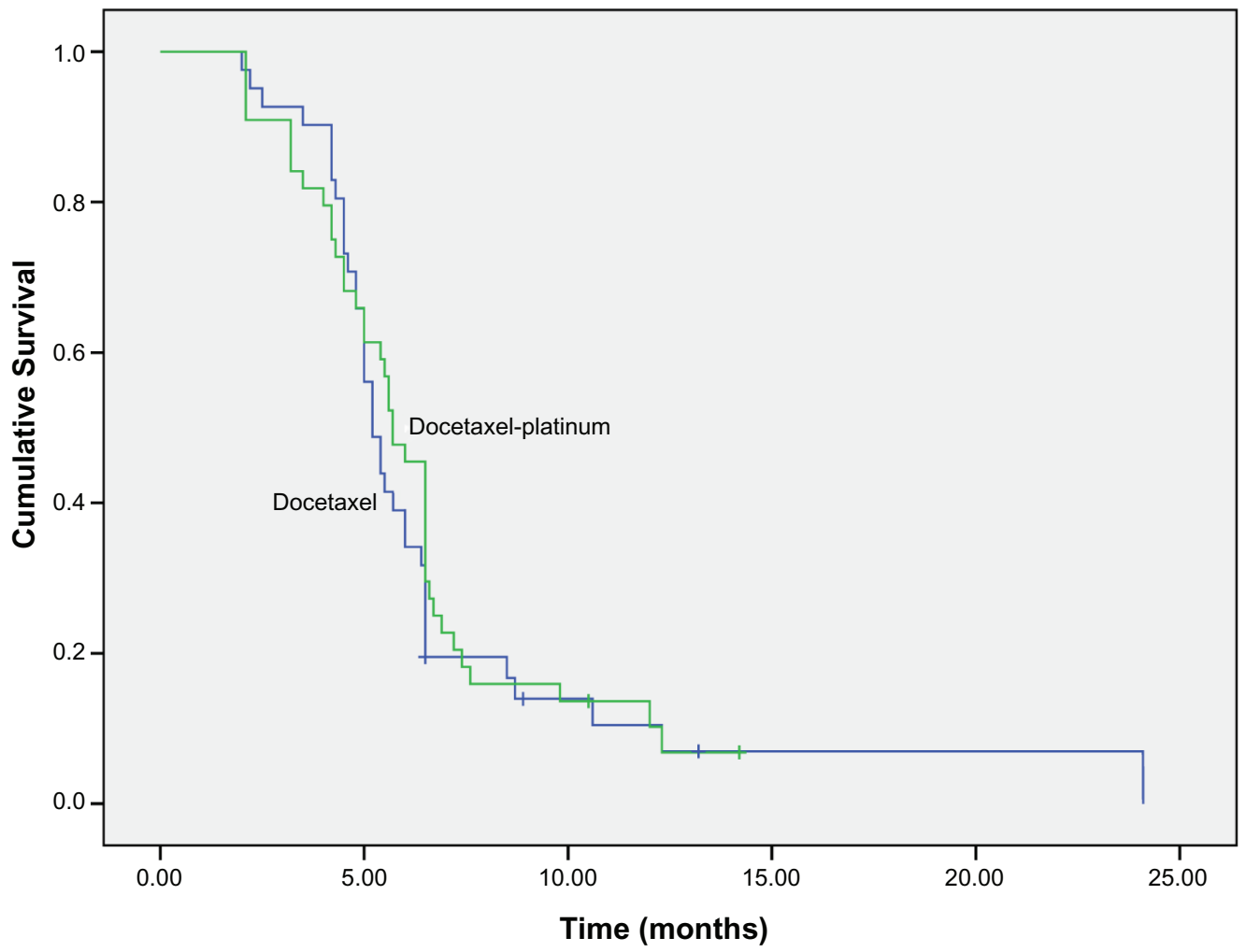

Figure 2 OS in docetaxel-platinum and docetaxel single-agent treatments group (5.7 vs 5.2 months, $P=0.64$ ). Abbreviation: OS, overall survival. 
Table 2 Efficacy of docetaxel-platinum and docetaxel single-agent group

\begin{tabular}{llll}
\hline & $\begin{array}{l}\text { Docetaxel- } \\
\text { platinum group } \\
(\mathbf{n = 4 4 )}\end{array}$ & $\begin{array}{l}\text { Docetaxel } \\
\text { group }(\mathbf{n}=\mathbf{4} \mathbf{I})\end{array}$ & P-value \\
\hline Complete response & 0 & 0 & - \\
Partial response & 13 & 8 & - \\
Stable disease & 14 & 13 & - \\
Progressive disease & 17 & 20 & - \\
Response rate & $29.5 \%$ & $19.5 \%$ & 0.28 \\
$\begin{array}{l}\text { Disease control rate } \\
\text { Median progression- }\end{array}$ & $61.4 \%$ & $51.2 \%$ & 0.35 \\
free survival & 3.6 months & 3.2 months & 0.38 \\
$\begin{array}{l}\text { Median overall } \\
\text { survival }\end{array}$ & 5.7 months & 5.2 months & 0.64 \\
\hline
\end{tabular}

accounting for overall response rate (ORR) and DCR of $24.7 \%$ and $56.5 \%$, respectively. Median PFS during secondline treatment was 3.5 months (95\% CI: 3.2-3.8). The median overall survival (OS) for all patients was 5.5 months (95\% CI: 4.1-6.2). There were no significant difference in ORR, DCR, PFS, and OS between docetaxel-platinum chemotherapy and docetaxel single-agent treatment $(P$-values were 0.38 and 0.64 , respectively) (Figures 1 and 2, and Table 2).

All 85 patients received fluorouracil-based first-line treatment. Forty patients had PR or complete response (CR) with first-line treatment, 45 patients with SD or progressive disease (PD). There was a significant difference in second-line PFS of patients who responded to first-line treatment $(\mathrm{PR}+\mathrm{CR})$ compared with nonresponding patients $(\mathrm{SD}+\mathrm{PD})$ (3.6 months vs 2.2 months, $P=0.005$ ) (Figure 3 ).

\section{Factors affecting PFS and OS by uni- and multivariate analyses}

Univariate analyses were performed by the Kaplan-Meier method to assess the predictive capability of each variable influencing PFS of second-line treatment. These data are summarized in Table 3. Sex, age, chemotherapy regimen (single-agent or combination treatment) were not found to be statistically associated with PFS in second-line treatment. Performance status score (PS) $(P=0.001)$ and response to first-line treatment $(P=0.02)$ were predictive of PFS (Table 3).

A multivariate Cox regression model was constructed with the incorporation of sex, age, PS chemotherapy regimen, and response to first-line treatment to evaluate the PFS and OS from the start of second-line treatment. PS $(P=0.024)$ and response to first-line treatment $(P=0.028)$ remained independent prognostic factors for PFS. PS had a significant influence on OS in multivariate analysis $(P=0.009)$ (Table 4$)$.

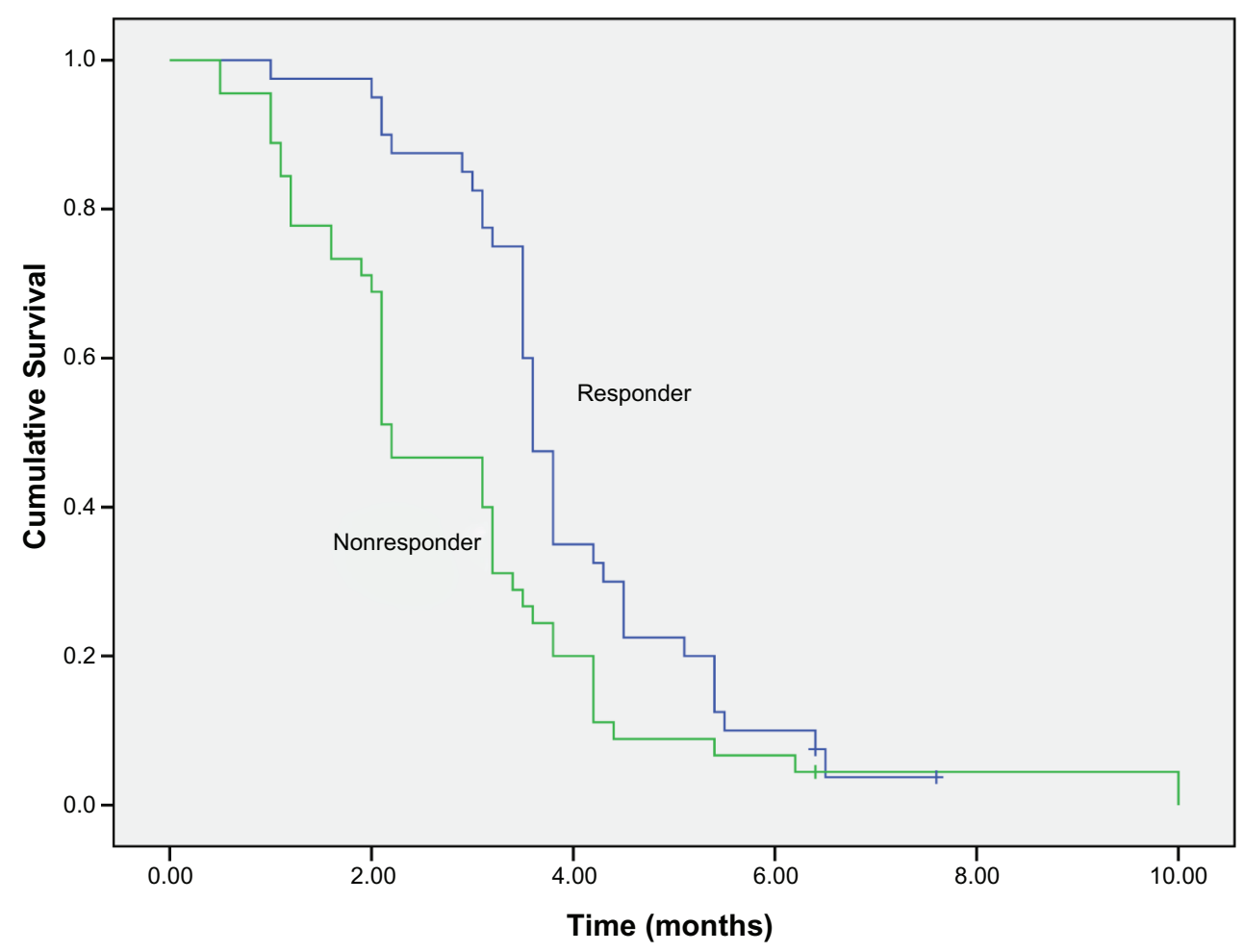

Figure 3 PFS of second-line docetaxel-based treatment according to first-line efficacy ( 3.6 vs 2.2 months, $P=0.005$ ).

Abbreviation: PFS, progression-free survival. 
Table 3 Univariate analysis of PFS and OS in second-line docetaxel-based treatment

\begin{tabular}{|c|c|c|c|c|c|c|}
\hline & Median PFS (months) & $95 \% \mathrm{Cl}$ & $P$-value & Median OS (months) & $95 \% \mathrm{Cl}$ & $P$-value \\
\hline Sex & & & 0.67 & & & 0.86 \\
\hline Male & 3.5 & $3.2-3.8$ & & 5.6 & $5.2-6.0$ & \\
\hline Female & 2.2 & $0.4-4.0$ & & 4.3 & $3.9-4.7$ & \\
\hline Age & & & 0.10 & & & 0.11 \\
\hline$<60$ & 3.5 & $3.2-3.8$ & & 5.6 & $5.0-6.2$ & \\
\hline$\geq 60$ & 3.1 & $2.0-4.2$ & & 5.2 & $4.8-5.8$ & \\
\hline ECOG PS & & & 0.002 & & & 0.001 \\
\hline $0-1$ & 3.6 & $3.3-3.9$ & & 6.0 & $5.5-6.5$ & \\
\hline 2 & 3.2 & $3.0-3.4$ & & 4.8 & $4.0-5.6$ & \\
\hline Initial disease status & & & 0.76 & & & 0.77 \\
\hline Metastatic & 3.5 & $3.0-3.8$ & & 5.6 & $5.0-6.0$ & \\
\hline Relapsed & 3.2 & $3.0-3.5$ & & 5.4 & $5.2-5.8$ & \\
\hline Response to first-line treatment & & & 0.005 & & & 0.02 \\
\hline$C R+P R$ & 3.6 & $3.4-3.8$ & & 6.0 & $5.3-6.7$ & \\
\hline $\mathrm{SD}+\mathrm{PD}$ & 2.2 & $1.6-2.8$ & & 5.0 & $4.3-5.7$ & \\
\hline Chemotherapy & & & 0.38 & & & 0.64 \\
\hline Docetaxel-platinum & 3.6 & $3.2-4.0$ & & 5.7 & $5.2-6.2$ & \\
\hline Docetaxel & 3.2 & $2.7-3.7$ & & 5.2 & $4.7-5.7$ & \\
\hline
\end{tabular}

Abbreviations: $\mathrm{Cl}$, confidence interval; CR, complete response; ECOG PS, the Eastern Cooperative Oncology Group scale of Performance Status; OS, overall survival; PD, progressive disease; PFS, progression-free survival; PR, partial response; SD, stable disease.

\section{Toxicities of different regimen treatment}

All patients who received at least one dose of chemotherapy were evaluated for toxicities. Toxicities of the docetaxelbased regimen were manageable, and no treatment-related deaths were observed. The frequency of grade $3 / 4$ toxicities is summarized in Table 4. Dosage reduction occurred in eight patients (Table 5).

\section{Discussion}

The present retrospective analysis involved 85 patients treated with docetaxel-based chemotherapy for advanced ESCC. The results indicated a PFS of 3.5 months for the second-line treatment with docetaxel-based chemotherapy. To our knowledge, this is the largest report focusing on the efficacy of docetaxel-based regimen for second-line treatment of ESCC.

Several studies have reported on chemotherapy for advanced esophageal cancer; however, most of these reports evaluated a first-line regimen, and only small number of studies focused on second-line chemotherapy. ${ }^{6}$ Approximately $40 \%$ of advanced esophageal cancer patients who experience progressive disease after first-line chemotherapy are able to receive second-line treatment based on previous studies. ${ }^{6}$ On the basis of data published so far, most of the studies were retrospective studies with a limited number of patients; ${ }^{7-11}$ no standard second-line therapy has emerged until now.

Docetaxel demonstrated good efficacy and is widely used in several solid tumors. More recently, these newer agents have been evaluated as a single agent or in combination in advanced settings for advanced ESCC as secondline chemotherapy. ${ }^{12-17}$ Among previous studies focusing on second-line chemotherapy, efficacy ranged from $0 \%$ to $50 \%$, with a PFS ranging from 3.3 to 6.5 months. OS was 5.1 to 11.4 months. ${ }^{6}$ However, no study compared efficacy of single-agent and combination chemotherapy for second-line treatment. In our cohort, there were no statistically significant differences in PFS and OS between the two groups in second-line treatment of advanced ESCC, which indicated

Table 4 Multivariate Cox regression analysis of PFS and OS in second-line treatments

\begin{tabular}{|c|c|c|c|c|c|c|}
\hline & \multicolumn{3}{|l|}{ PFS } & \multicolumn{3}{|l|}{ OS } \\
\hline & HR & $95 \% \mathrm{Cl}$ & $P$-value & HR & $95 \% \mathrm{Cl}$ & $P$-value \\
\hline Sex & 0.990 & $0.415-2.358$ & 0.990 & 1.005 & $0.387-2.612$ & 0.992 \\
\hline Age & 0.732 & $0.455-1.177$ & 0.198 & 0.645 & $0.396-1.049$ & 0.077 \\
\hline Response to first-line & 1.618 & $1.059-2.678$ & 0.028 & 1.500 & $0.928-2.425$ & 0.098 \\
\hline Performance score & 1.824 & I.083-3.072 & 0.024 & 1.957 & I.179-3.246 & 0.009 \\
\hline Second-line drug & $0.74 I$ & 0.469 & I.I7I & 0.866 & $0.550-1.365$ & 0.536 \\
\hline
\end{tabular}

Abbreviations: $\mathrm{Cl}$, confidence interval; $\mathrm{HR}$, hazard ratio; OS, overall survival; PFS, progression-free survival. 
Table 5 The main grade $3 / 4$ toxicity of docetaxel-platinum and docetaxel single-agent group

\begin{tabular}{lll}
\hline & $\begin{array}{l}\text { Docetaxel-platinum } \\
\text { group }(\mathbf{n = 4 4 )}\end{array}$ & $\begin{array}{l}\text { Docetaxel } \\
\text { group }(\mathbf{n = 4} \text { I) }\end{array}$ \\
\hline Nausea/vomiting & $6(13.6 \%)$ & $2(4.9 \%)$ \\
Alopecia & $8(18.2 \%)$ & $7(17.1 \%)$ \\
Diarrhea & $2(4.5 \%)$ & $0(0.0 \%)$ \\
Neurotoxicity & $3(6.8 \%)$ & $3(7.3 \%)$ \\
Constipation & $4(9.1 \%)$ & $2(4.9 \%)$ \\
Pyrexia & $6(13.6 \%)$ & $4(9.8 \%)$ \\
Thrombocytopenia & $6(13.6 \%)$ & $3(7.3 \%)$ \\
Neutropenia & $15(34.1 \%)$ & $9(22.0 \%)$ \\
Anemia & $5(11.4 \%)$ & $3(7.3 \%)$ \\
Dosage reduction & $6(13.6 \%)$ & $2(4.9 \%)$ \\
\hline
\end{tabular}

that single-agent treatment is enough for second-line chemotherapy of ESCC.

With no prospective trials and a limited number of retrospective studies, no study has been conducted to identify the prognostic factors in second-line chemotherapy of ESCC. The present study demonstrated that response to first-line chemotherapy along with PS were associated with a significantly better prognosis.

The major limitation in the current study is its retrospective nature. In addition, the small sample size of cases may not be sufficient to accurately interpret the study results. Further assessment in a randomized, prospective study is required to obtain definitive evidence.

\section{Conclusion}

The results of this study showed a moderate PFS advantage with docetaxel-based treatment in second-line chemotherapy of ESCC. There were no efficacy differences between docetaxel single-agent and combination regimens. Response to first-line chemotherapy was an important prognostic factor for PFS of second-line chemotherapy. Further prospective studies are warranted to elucidate any potential efficacy and toxicity of the docetaxel-based regimen.

\section{Disclosure}

The authors report no conflicts of interest in this work.

\section{References}

1. Siegel R, Naishadham D, Jemal A. Cancer statistics. CA Cancer J Clin. 2013;63(1):11-30.
2. Yang L, Parkin DM, Li L, Chen Y. Time trends in cancer mortality in China: 1987-1999. Int J Cancer. 2003;106(5):771-783.

3. Udagawa H. Chemoradiotherapy: its effectiveness, toxicity, and perspective in the treatment of esophageal cancer. Ann Thorac Cardiovasc Surg. 2009;15(6):359-361.

4. Ajani JA. Contributions of chemotherapy in the treatment of carcinoma of the esophagus: results and commentary. Semin Oncol. 1994;21(4):474-482.

5. Ford HE, Marshall A, Bridgewater JA, et al; COUGAR-02 Investigators. Docetaxel versus active symptom control for refractory oesophagogastric adenocarcinoma (COUGAR-02): an open-label, phase 3 randomised controlled trial. Lancet Oncol. 2014;15(1):78-86.

6. Thallinger CM, Raderer M, Hejna M. Esophageal cancer: a critical evaluation of systemic second-line therapy. J Clin Oncol. 2011;29(35):4709-4714.

7. Jin J, Xu X, Wang F, et al. Second-line combination chemotherapy with docetaxel and nedaplatin for Cisplatin-pretreated refractory metastatic/recurrent esophageal squamous cell carcinoma. $J$ Thorac Oncol. 2009;4(8):1017-1021.

8. Tanaka T, Fujita H, Sueyoshi S, et al. Second-line combination chemotherapy with docetaxel for cisplatin-pretreated refractory metastatic esophageal cancer: a preliminary report of initial experience. Chemotherapy. 2007;53(6):449-453.

9. Kanai M, Matsumoto S, Nishimura T, et al. Retrospective analysis of 27 consecutive patients treated with docetaxel/nedaplatin combination therapy as a second-line regimen for advanced esophageal cancer. Int J Clin Oncol. 2007;12(3):224-227.

10. Osaka Y, Takagi Y, Hoshino S, Tachibana S, Tsuchida A, Aoki T. Combination chemotherapy with docetaxel and nedaplatin for recurrent esophageal cancer in an outpatient setting. Dis Esophagus. 2006;19(6):473-476.

11. Yoshioka T, Sakayori M, Kato S, et al. Dose escalation study of docetaxel and nedaplatin in patients with relapsed or refractory squamous cell carcinoma of the esophagus pretreated using cisplatin, 5-fluorouracil, and radiation. Int J Clin Oncol. 2006;11(6):454-460.

12. Muro K, Hamaguchi T, Ohtsu A, et al. A phase II study of single-agent docetaxel in patients with metastatic esophageal cancer. Ann Oncol. 2004;15(6):955-959.

13. Nakajima Y, Suzuki T, Haruki S, et al. A pilot trial of docetaxel and nedaplatin in cisplatin-pretreated relapsed or refractory esophageal squamous cell cancer. Hepatogastroenterology. 2008;55(86-87):1631-1635.

14. Anderson SE, O'Reilly EM, Kelsen DP, Ilson DH. Phase II trial of 96-hour paclitaxel in previously treated patients with advanced esophageal cancer. Cancer Invest. 2003;21(4):512-516.

15. Lordick F, von Schilling C, Bernhard H, Hennig M, Bredenkamp R, Peschel C. Phase II trial of irinotecan plus docetaxel in cisplatinpretreated relapsed or refractory oesophageal cancer. $\mathrm{Br} J$ Cancer. 2003;89(4):630-633.

16. Conroy T, Etienne PL, Adenis A, et al. Phase II trial of vinorelbine in metastatic squamous cell esophageal carcinoma. European Organization for Research and Treatment of Cancer Gastrointestinal Treat Cancer Cooperative Group. J Clin Oncol. 1996;14(1):164-170.

17. Wang M, Gu J, Wang HX, Wu MH, Li YM, Wang YJ. Retrospective study of gemcitabine based chemotherapy for unresectable or recurrent esophagus squamous cell carcinoma refractory to first line chemotherapy. Asian Pac J Cancer Prev. 2012;13(8):4153-4156. 
OncoTargets and Therapy

\section{Publish your work in this journal}

OncoTargets and Therapy is an international, peer-reviewed, open access journal focusing on the pathological basis of all cancers, potential targets for therapy and treatment protocols employed to improve the management of cancer patients. The journal also focuses on the impact of management programs and new therapeutic agents and protocols on

patient perspectives such as quality of life, adherence and satisfaction. The manuscript management system is completely online and includes a very quick and fair peer-review system, which is all easy to use. Visit http://www.dovepress.com/testimonials.php to read real quotes from published authors.

Submit your manuscript here: http://www.dovepress.com/oncotargets-and-therapy-journal 